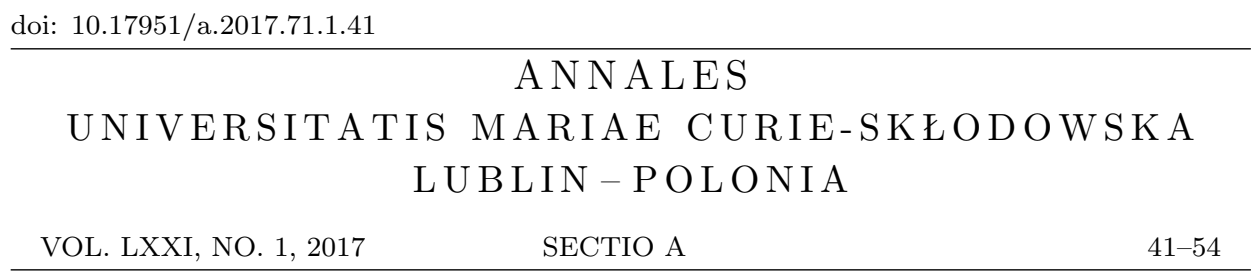

DOMINIKA JASIŃSKA

\title{
A spatial individual-based contact model with age structure
}

\begin{abstract}
The Markov dynamics of an infinite continuum birth-and-death system of point particles with age is studied. Each particle is characterized by its location $x \in \mathbb{R}^{d}$ and age $a_{x} \geq 0$. The birth and death rates of a particle are age dependent. The states of the system are described in terms of probability measures on the corresponding configuration space. The exact solution of the evolution equation for the correlation functions of first and second orders is found.
\end{abstract}

1. Introduction. We describe the Markov evolution of a continuum infinite system of particles with an age structure. An infinite continuum particle system can provide a good model for the evolution of atoms, dust grains, water droplets and molecules. Such models with an age structure can describe stellar systems, like galaxies, or large communities of infected individuals. The most important facts on the approach we follow in this work can be found in $[1,4,7,12]$. In this approach, the states of the system are probability measures on the corresponding configuration spaces, the Markov evolution of which is obtained by solving a Fokker-Planck equation. In contrast to the works just cited, we consider a system with an age structure, and therefore employ so-called marked configurations. To the best of our knowledge this is the first attempt in studying infinite systems of this type. Finite systems of particles with age were studied in $[10,9]$. In this

2010 Mathematics Subject Classification. 60K35, 60G55, 35Q70, 82C22, 92D25.

Key words and phrases. Correlation function, contact model, birth and death model, configuration space, spatial individual-based model, Markov evolution, age structure. 
work, the exact formulas describing the Markov evolution of the first and second correlation functions are obtained.

2. The mathematical preliminaries. We begin by introducing the basic concepts and notions.

Definition 2.1. The configuration space $\Gamma$ is

$$
\Gamma=\left\{\gamma \subset \mathbb{R}^{d}:|\gamma \cap A|<\infty \text { for any compact set } \mathrm{A} \subset \mathbb{R}^{d}\right\} .
$$

The set of all finite configurations is

$$
\Gamma_{0}=\bigcup_{n \in \mathbb{N}_{0}}\left\{\gamma \subset \mathbb{R}^{d}:|\gamma|=n\right\} .
$$

In our considerations, each particle is described by its location $x \in \mathbb{R}^{d}$ and age $a_{x} \geq 0$, that is, by $\hat{x}=\left(x, a_{x}\right)$. For a $\gamma \in \Gamma$, by $\hat{\gamma}$ we denote the marked configuration $\left\{\hat{x}=\left(x, a_{x}\right): x \in \gamma\right\}$. In this case, $\gamma$ is the underlying configuration for $\hat{\gamma}$. By $p$ we denote the corresponding projection, i.e., the map $\hat{\gamma} \mapsto \gamma=p(\hat{\gamma})$. This can also be defined in the following way. Set $\hat{X}=\mathbb{R}^{d} \times \mathbb{R}_{+}, \mathbb{R}_{+}:=[0,+\infty)$. Let $p: \hat{X} \rightarrow \mathbb{R}^{d}$ be the projection $p(\hat{x})=x$ for $\hat{x}=\left(x, a_{x}\right)$. This map can naturally be extended to subsets of $\hat{X}$ to give the projection meant above. That is, for $\hat{A} \subset \hat{X}, p(\hat{A})=\{p(\hat{x}): \hat{x} \in \hat{A}\}$.

Definition 2.2. The space of marked configurations is

$$
\hat{\Gamma}=\{\hat{\gamma} \subset \hat{X}: p(\hat{\gamma}) \in \Gamma\},
$$

where $\Gamma$ is as in Definition 2.1.

Let $f: \hat{X} \rightarrow \mathbb{R}$ be a continuous function with compact support with respect to $x$ and continuous with respect to $a$. The space $\hat{\Gamma}$ is equipped with the vague topology - the weakest topology that makes continuous the maps

$$
\hat{\gamma} \rightarrow \sum_{x \in p_{X}(\hat{\gamma})} f(\hat{x}) \in \mathbb{R} .
$$

This topology is separably and completely metrizable, see [3]. Then $\hat{\Gamma}$ with the corresponding Borel $\sigma$-field $\mathcal{B}(\hat{\Gamma})$ gets a standard Borel space. The set of all probability measures on $(\hat{\Gamma}, \mathcal{B}(\hat{\Gamma}))$ is denoted by $\mathcal{P}(\hat{\Gamma})$.

The evolution of the model we consider is described by a Markov generator $L$, which acts on observables $F: \hat{\Gamma} \rightarrow \mathbb{R}$, being appropriate measurable functions. The value of an observable at state $\mu$ is given by

$$
<F, \mu>=\int_{\hat{\Gamma}} F(\hat{\gamma}) d \mu(\hat{\gamma}) .
$$

Our generator satisfies the backward Kolmogorov equation

$$
\frac{\partial}{\partial t} F_{t}=L F_{t}
$$


As is usual, when dealing with infinite systems one employs correlation functions, see $[1,4,6,7,12]$ for more detail. This supposes to pass to functions defined on the set of finite configurations $\hat{\Gamma}_{0}:=\{\hat{\gamma} \in \hat{\Gamma}:|p(\hat{\gamma})|<$ $\infty$. Since $\hat{\Gamma}_{0}$ is in $\mathcal{B}(\hat{\Gamma})$, it can be equipped with the induced $\sigma$-field $\mathcal{B}\left(\hat{\Gamma}_{0}\right)$.

Definition 2.3. The Lebesgue-Poisson measure on $\left(\hat{\Gamma}_{0}, \mathcal{B}\left(\hat{\Gamma}_{0}\right)\right)$ is determined by the expression, see [8],

$$
\hat{\lambda}_{\sigma}=\sum_{n=0}^{\infty} \frac{\sigma^{(n)}}{n !}
$$

where $\sigma^{(n)}\left(d \hat{x}_{1}, \ldots, d \hat{x}_{n}\right)=d \hat{x}_{1} \ldots d \hat{x}_{n}, d \hat{x}_{i}:=d x_{1} d a_{x_{i}}$.

Let $G \in \mathcal{B}_{b s}(\hat{\Gamma})$ be the set of all measurable functions $G: \hat{\Gamma} \rightarrow \mathbb{R}$ such that $G(\hat{\gamma})=0$ whenever $\gamma=p(\hat{\gamma})$ is not contained in a certain compact $\Lambda$ or $|\gamma|>N$, where $\Lambda$ and $N \in \mathbb{N}$ are specific for this $G$. It is clear that each such a function is supported on $\hat{\Gamma}_{0}$.

Definition 2.4. Let $\mu$ be a probability measure on $(\hat{\Gamma}, \mathcal{B}(\hat{\Gamma}))$. If, for some measurable $k_{\mu}: \hat{\Gamma}_{0} \rightarrow \mathbb{R}$ and all $G \in \mathcal{B}_{b s}(\hat{\Gamma})$, the equality

$$
\int_{\hat{\Gamma}} \sum_{\eta \Subset \gamma} G(\hat{\eta}) d \mu(\hat{\gamma})=\int_{\hat{\Gamma}_{0}} G(\hat{\eta}) k_{\mu}(\hat{\eta}) d \hat{\lambda}(\hat{\eta})
$$

holds, then $k_{\mu}: \hat{\Gamma}_{0} \rightarrow \mathbb{R}$ is called a correlation function for $\mu$. Here $\sum_{\eta \Subset \gamma}$ denotes the sum taken over finite sub-configurations of $\gamma$.

Now we introduce a function $F_{\theta}(\hat{\gamma})$ by the formula

$$
F_{\theta}(\hat{\gamma})=\prod_{y \in \gamma}(1+\theta(\hat{y}))
$$

where $\theta(\hat{x}) \in(-1,0]$ is a $\mathcal{B}\left(\mathbb{R}^{d}, \mathbb{R}_{+}\right)$-measurable bounded function with compact support. For a measure $\mu \in \mathcal{P}(\hat{\Gamma})$, the Bogoliubov functional is defined by

$$
B_{\mu}(\theta)=\int_{\hat{\Gamma}} F_{\theta}(\hat{\gamma}) \mu(d \hat{\gamma})
$$

In our considerations we employ the measures $\mu$ such that $B_{\mu}$ can be continued to a function of $\theta \in\left(L^{1}\left(\mathbb{R}^{d}\right), L^{\infty}\left(\mathbb{R}_{+}\right)\right)$which is analytic on some neighborhood of $\theta=0$. Then (2.4) can be written in the following form, see $[2]$,

$$
\begin{aligned}
B_{\mu}(\theta) & =1+\sum_{n=1}^{\infty} \frac{1}{n !} \int_{\hat{X}^{n}} k_{\mu}{ }^{(n)}\left(\hat{x}_{1}, \ldots, \hat{x}_{n}\right) \theta\left(\hat{x}_{1}\right) \ldots \theta\left(\hat{x}_{n}\right) d \hat{x}_{1} \ldots d \hat{x}_{n} \\
& =\int_{\hat{\Gamma}_{0}} k_{\mu}(\hat{\eta}) \prod_{x \in \eta} \theta(\hat{x}) \lambda(d \hat{\eta}),
\end{aligned}
$$


where each $k_{\mu}{ }^{(n)}$ is a symmetric measurable function $\hat{X}^{n} \rightarrow \mathbb{R}$, called $n$-th order correlation function. It can also be defined as a restriction of $k_{\mu}$, i.e., $k_{\mu}{ }^{(n)}\left(\hat{x}_{1}, \ldots, \hat{x}_{n}\right)=k_{\mu}(\hat{\eta})$ for $\hat{\eta}=\left\{\hat{x}_{1}, \ldots, \hat{x}_{n}\right\}$. In addition to what was assumed above regarding the measure $\mu$, we also will assume that its correlation function satisfies:

(i) $\lim _{a_{\hat{\eta}}^{+} \rightarrow+\infty} k_{\mu}(\hat{\eta})=0, \quad a_{\hat{\eta}}^{+}:=\max _{x \in \eta} a_{x}$

(ii) $k_{\mu}{ }^{(n)}$ is in $L^{\infty}\left(\mathbb{R}^{d}\right)$ with respect to each of $x_{1}, \ldots, x_{n}$ and in $C^{1}([0, \infty)) \cap L^{1}([0, \infty))$ with respect to each of $a_{1}, \ldots, a_{n}$. That is, for each $n \in \mathbb{N}$, we have that

$$
\int_{\left(\mathbb{R}_{+}\right)^{n}} k_{0}^{(n)}\left(\hat{x}_{1}, \ldots, \hat{x}_{n}\right) d a_{1} \ldots d a_{n} \in L^{\infty}\left(\left(\mathbb{R}^{d}\right)^{n}\right) .
$$

Using the Bogoliubov functional, we can get across from computation in $\hat{\Gamma}$ to $\hat{\Gamma}_{0}$

$$
\int_{\hat{\Gamma}}\left(L F_{\theta}\right)(\hat{\gamma}) \mu(d \hat{\gamma})=\int_{\hat{\Gamma}_{0}}\left(L^{\triangle} k_{\mu}\right)(\hat{\eta}) \prod_{x \in \eta} \theta(\hat{x}) \lambda(d \hat{\eta}) .
$$

Then instead of (2.1) we will consider the Cauchy problem

$$
\left\{\begin{array}{l}
\frac{d}{d t} k_{t}=L^{\Delta} k_{t}, \\
\left.k_{t}\right|_{t=0}=k_{\mu_{0}} .
\end{array}\right.
$$

We conclude this part by presenting some formulas which will be used in the sequel:

$$
\begin{gathered}
\prod_{x \in \gamma}(1+\theta(\hat{x}))=\sum_{\eta \subset \gamma} \prod_{x \in \eta} \theta(\hat{x}), \\
\sum_{x \in \gamma} A(\hat{x}) \sum_{\eta \subset \gamma \backslash x} B(\hat{\eta})=\sum_{\eta \subset \gamma} \sum_{x \in \eta} A(\hat{x}) B(\hat{\eta} \backslash \hat{x}) .
\end{gathered}
$$

Lemma 2.5 (Minlos [11]). Let $n \in \mathbb{N}, n \geq 2$, then for all measurable functions $G: \hat{\Gamma}_{0} \rightarrow \mathbb{R}, H: \hat{\Gamma}_{0} \rightarrow \mathbb{R}$ it is true that:

$$
\int_{\hat{\Gamma}_{0}} \sum_{x \in \gamma} H(\hat{x}) G(\hat{\gamma} \backslash \hat{x}) \hat{\lambda}(d \hat{\gamma})=\int_{\hat{\Gamma}_{0}} \int_{\mathbb{R}^{d}} H(\hat{x}) G(\hat{\gamma}) d x \hat{\lambda}(d \hat{\gamma}) .
$$

3. The equation for the correlation functions. The model which we consider is described by means of the following Markov generator

$$
\begin{aligned}
(L F)(\hat{\gamma})= & \sum_{x \in \gamma} \frac{\partial}{\partial a_{x}} F(\hat{\gamma})+\sum_{x \in \gamma} m(\hat{x})[F(\hat{\gamma} \backslash \hat{x})-F(\hat{\gamma})] \\
& +\int_{\widehat{X}} \sum_{y \in \gamma} \delta\left(a_{x}\right) b(\hat{y} \mid x)[F(\hat{\gamma} \cup \hat{x})-F(\hat{\gamma})] d \hat{x} .
\end{aligned}
$$


The first summand in (3.1) describes the aging, cf. [9]. The second one describes the mortality with age and location dependent rate $m(\hat{x}) \geq 0$, whereas the third summand corresponds to the birth of a particle. It can also be interpreted as an infection spreading process in the population. In that case, $\hat{\gamma}$ is the configuration of infected individuals containing information on their location and the duration of illness. The recovery is described by the coefficient $m(\hat{x})$, whereas $b(\hat{y} \mid x)$ is the infection rate. In $[10,9]$, similar models were studied with the consideration restricted to finite systems. Set

$$
M(\hat{\eta})=\sum_{x \in \eta} m(\hat{x}) .
$$

Regarding the birth rate we will assume that

$$
\forall_{a \geq 0} \quad b(y, a \mid x) \leq \beta(x-y), \quad \int_{\mathbb{R}^{d}} \beta(x) d x=: \beta<\infty .
$$

Furthermore, we assume that $b(y, a \mid x)=0$ whenever $a \leq a_{0}$ for some $a_{0}>0$, and also

$$
\underset{x \in \mathbb{R}^{d}}{\operatorname{ess} \int_{\mathbb{R}^{d}}} \int_{0}^{\infty} b(y, a \mid x) d y d a:=\bar{b}<\infty .
$$

Proposition 3.1. The equation (2.6) with the initial condition $\left.k_{t}\right|_{t=0}=k_{0}$ and the renewal condition

$$
k_{t}(\hat{\eta} \backslash \hat{x} \cup(x, 0))=\int_{\widehat{X}} b(\hat{y} \mid x) k_{t}(\hat{\eta} \backslash \hat{x} \cup \hat{y}) d \hat{y}+k_{t}(\hat{\eta} \backslash \hat{x}) \sum_{y \in \eta \backslash x} b(\hat{y} \mid x)
$$

takes the form

$$
\frac{\partial}{\partial t} k_{t}(\hat{\eta})=-\sum_{x \in \eta} \frac{\partial}{\partial a_{x}} k_{t}(\hat{\eta})-M(\hat{\eta}) k_{t}(\hat{\eta}) .
$$

Proof. By means of Lemma 2.5 and (2.8) the operator $L$ given in (3.1) can be transformed, cf. (2.5), to the following

$$
\begin{aligned}
& \left(L^{\Delta} k_{\mu}\right)(\hat{\eta})=-\sum_{x \in \eta} \frac{\partial}{\partial a_{x}} k_{\mu}(\hat{\eta})-M(\hat{\eta}) k_{\mu}(\hat{\eta}) \\
& +\sum_{x \in \eta} \delta\left(a_{x}\right)\left[-k_{\mu}(\hat{\eta})+\int_{\widehat{X}} b(\hat{y} \mid x) k_{\mu}(\hat{\eta} \backslash \hat{x} \cup \hat{y}) d \hat{y}+k_{\mu}(\hat{\eta} \backslash \hat{x}) \sum_{y \in \eta \backslash x} b(\hat{y} \mid x)\right] .
\end{aligned}
$$

The action of the generator (3.1) on $F_{\theta}(2.3)$ can be written as

$$
\begin{aligned}
L F_{\theta}(\hat{\gamma})= & \sum_{x \in \gamma} \frac{\partial \theta(\hat{x})}{\partial a_{x}} \prod_{y \in \gamma \backslash x}(1+\theta(\hat{y}))+\sum_{x \in \gamma} m(\hat{x})(1-(1+\theta(\hat{x}))) \prod_{y \in \gamma \backslash x}(1+\theta(\hat{y})) \\
& +\int_{\hat{X}} \sum_{x \in \gamma} b(\hat{x} \mid \hat{y}) \delta\left(a_{y}\right)(1+\theta(\hat{y})-1) \prod_{z \in \gamma}(1+\theta(\hat{z})) d \hat{y} .
\end{aligned}
$$


Now we split this into three parts. The first part is

$$
\begin{aligned}
\int_{\hat{\Gamma}} L_{1} F_{\theta}(\hat{\gamma}) \mu(d \hat{\gamma})= & \int_{\hat{\Gamma}} \sum_{x \in \gamma} \frac{\partial \theta(\hat{x})}{\partial a_{x}} \prod_{y \in \gamma \backslash x}(1+\theta(\hat{y})) \mu(d \hat{\gamma}) \\
= & \int_{\hat{\Gamma}} \sum_{x \in \gamma} \frac{\partial \theta(\hat{x})}{\partial a_{x}} \sum_{\eta \subset \gamma \backslash x} \prod_{y \in \eta} \theta(\hat{y}) \mu(d \hat{\gamma}) \\
= & \int_{\hat{\Gamma}} \sum_{\eta \subset \gamma} \sum_{x \in \eta} \frac{\partial \theta(\hat{x})}{\partial a_{x}} \prod_{y \in \eta \backslash x} \theta(\hat{y}) \mu(d \hat{\gamma}) \\
= & \int_{\hat{\Gamma}_{0}} k_{\mu}(\hat{\eta}) \sum_{x \in \gamma} \frac{\partial \theta(\hat{x})}{\partial a_{x}} \prod_{y \in \eta \backslash x} \theta(\hat{y}) d \hat{x} \lambda(d \hat{\eta}) \\
= & \int_{\hat{\Gamma}_{0}}\left(\int_{\hat{X}} k_{\mu}(\hat{\eta} \cup \hat{x}) \frac{\partial \theta(\hat{x})}{\partial a_{x}} d \hat{x}\right) \prod_{y \in \eta} \theta(\hat{y}) \lambda(d \hat{\eta}) \\
= & -\int_{\hat{\Gamma}_{0}}\left(\int_{\hat{X}} k_{\mu}(\hat{\eta} \cup \hat{x}) \delta\left(a_{x}\right)\right. \\
& \left.+\frac{\partial}{\partial a_{x}} k_{\mu}(\hat{\eta} \cup \hat{x}) \theta(\hat{x}) d \hat{x}\right) \prod_{y \in \eta} \theta(\hat{y}) \lambda(d \hat{\eta}) \\
= & -\int_{\hat{\Gamma}_{0}}\left(\sum_{x \in \eta} \delta\left(a_{x}\right) k_{\mu}(\hat{\eta})+\frac{\partial}{\partial a_{x}} k_{\mu}(\hat{\eta}) \theta(\hat{x})\right) \prod_{y \in \eta} \theta(\hat{y}) \lambda(d \hat{\eta})
\end{aligned}
$$

This result is obtained by using $(2.7),(2.8)$ with $A(x)=\frac{\partial \theta(\hat{x})}{\partial a_{x}}, B(\hat{\eta})=$ $\prod_{y \in \eta} \theta(\hat{y}),(2.2)$ and Minlos' lemma (2.9). Similarly, we get the next two parts. The second part

$$
\begin{aligned}
\int_{\hat{\Gamma}} L_{2} F_{\theta}(\hat{\gamma}) \mu(d \hat{\gamma}) & =\int_{\hat{\Gamma}} \sum_{x \in \gamma}(-\theta(\hat{x})) m(\hat{x}) \prod_{y \in \gamma \backslash x}(1+\theta(\hat{y})) \mu(d \hat{\gamma}) \\
& =-\int_{\hat{\Gamma}} \sum_{x \in \gamma} \theta(\hat{x}) m(\hat{x}) \sum_{\eta \subset \gamma \backslash x} \prod_{y \in \eta} \theta(\hat{y}) \mu(d \hat{\gamma}) \\
& =-\int_{\hat{\Gamma}} \sum_{\eta \subset \gamma} \sum_{x \in \eta} m(\hat{x}) \prod_{y \in \eta} \theta(\hat{y}) \mu(d \hat{\gamma}) \\
& =-\int_{\hat{\Gamma_{0}}} \sum_{x \in \eta} m(\hat{x}) k_{\mu}(\hat{\eta}) \prod_{y \in \eta} \theta(\hat{y}) \lambda(d \hat{\eta}) .
\end{aligned}
$$

The third part

$$
\int_{\hat{\Gamma}} L_{3} F_{\theta}(\hat{\gamma}) \mu(d \hat{\gamma})=\int_{\hat{\Gamma}} \int_{\hat{X}} \sum_{x \in \gamma} b(\hat{x} \mid \hat{y}) \delta\left(a_{y}\right) \theta(\hat{y}) \prod_{z \in \gamma}(1+\theta(\hat{z})) d \hat{y} \mu(d \hat{\gamma})
$$




$$
\begin{aligned}
= & \left.\int_{\hat{\Gamma}} \int_{\hat{X}} \sum_{x \in \gamma} b(\hat{x} \mid \hat{y}) \delta\left(a_{y}\right) \theta(\hat{y})(1+\theta(\hat{x}))\right) \\
& \times \prod_{z \in \gamma \backslash x}(1+\theta(\hat{z})) d \hat{y} \mu(d \hat{\gamma}) \\
= & \int_{\hat{\Gamma}} \int_{\hat{X}} \sum_{x \in \gamma} b(\hat{x} \mid \hat{y}) \delta\left(a_{y}\right) \theta(\hat{y})(1+\theta(\hat{x})) \\
& \times \sum_{\eta \subset \gamma \backslash x} \prod_{z \in \eta} \theta(\hat{z}) d \hat{y} \mu(d \hat{\gamma}) \\
= & \int_{\hat{\Gamma}} \int_{\hat{X}} \sum_{\eta \subset \gamma} \sum_{x \in \eta} b(\hat{x} \mid \hat{y}) \delta\left(a_{y}\right)(1+\theta(\hat{x})) \\
& \times \prod_{z \in \eta \backslash x \cup y} \theta(\hat{z}) d \hat{y} \mu(d \hat{\gamma}) \\
= & \int_{\hat{\Gamma}_{0}} \int_{\hat{X}} k_{\mu}(\hat{\eta}) \sum_{x \in \eta} b(\hat{x} \mid \hat{y}) \delta\left(a_{y}\right) \prod_{z \in \eta \backslash x \cup y} \theta(\hat{z}) d \hat{y} \lambda(d \hat{\eta}) \\
& +\int_{\hat{\Gamma}_{0}} \int_{\hat{X}} k_{\mu}(\hat{\eta}) \sum_{x \in \eta} b(\hat{x} \mid \hat{y}) \delta\left(a_{y}\right) \prod_{z \in \eta \cup y} \theta(\hat{z}) d \hat{y} \lambda(d \hat{\eta}) \\
= & \int_{\hat{\Gamma}_{0}} \int_{\hat{X}} \sum_{y \in \eta} k_{\mu}(\hat{\eta} \cup \hat{x} \backslash \hat{y}) b(\hat{x} \mid \hat{y}) \delta\left(a_{y}\right) \prod_{z \in \eta} \theta(\hat{z}) d \hat{x} \lambda(d \hat{\eta}) \\
& +\int_{\hat{\Gamma}_{0}} \int_{\hat{X}} \sum_{y \in \eta} k_{\mu}(\hat{\eta} \backslash \hat{y}) b(\hat{x} \mid \hat{y}) \delta\left(a_{y}\right) \prod_{z \in \eta} \theta(\hat{z}) d \hat{y} \lambda(d \hat{\eta}) .
\end{aligned}
$$

Then by (2.5) we obtain (3.5). To cancel the last part of the latter we use the renewal condition (3.3), cf. [5]. Thereafter, we arrive at (3.4).

To solve (3.4) we use the method of characteristics by means of which it can be transformed to the ordinary differential equation.

Set

$$
\psi_{\tau}(\hat{\eta})=k_{t-\tau}^{(n)}\left(\hat{\eta}_{\tau}\right)
$$

where

$$
\hat{\eta}_{\tau}=\left\{\left(x, a_{x}-\tau\right):\left(x, a_{x}\right) \in \hat{\eta}\right\}, \quad \tau=\min \left\{a_{x}, t\right\} .
$$

Then

$$
\frac{d}{d \tau} \psi_{\tau}(\hat{\eta})=: \dot{\psi}_{\tau}(\hat{\eta})=M\left(\hat{\eta}_{\tau}\right) \psi_{\tau}(\hat{\eta})
$$

which yields

$$
\psi_{\tau}(\hat{\eta})=\psi_{0}(\hat{\eta}) \exp \left(\int_{0}^{\tau} M\left(\hat{\eta}_{\theta}\right) d \theta\right)
$$


Then we apply this in (3.6) to get, where $a_{\hat{\eta}}=\min _{x \in \eta} a_{x}$

$$
k_{t}^{(n)}(\hat{\eta})= \begin{cases}k_{0}^{(n)}\left(\hat{\eta}_{t}\right) \exp \left(-\int_{0}^{t} M\left(\hat{\eta}_{\theta}\right) d \theta\right), & t \leq a_{\hat{\eta}} \\ k_{t-a_{\hat{\eta}}}{ }^{(n)}\left(\hat{\eta}_{a_{\hat{\eta}}}\right) \exp \left(-\int_{0}^{a_{\hat{\eta}}} M\left(\hat{\eta}_{\theta}\right) d \theta\right), & t>a_{\hat{\eta}} .\end{cases}
$$

4. Solving the equation. The first line in (3.7) gives the solution of (3.4) for small $t$, whereas the second line is still an equation, which we are going to solve in this section for $n=1,2$.

4.1. The solution for $\boldsymbol{n}=\mathbf{1}$. For $n=1$, the equation in (3.7) turns into the following

$$
k_{t}^{(1)}(x, a)= \begin{cases}k_{0}{ }^{(1)}(x, a-t) \exp \left(-\int_{0}^{t} m(x, a-\theta) d \theta\right), & t \leq a \\ k_{t-a}{ }^{(1)}(x, 0) \exp \left(-\int_{0}^{a} m(x, a-\theta) d \theta\right), & t>a .\end{cases}
$$

By (3.3) we get

$$
k_{t}^{(1)}(x, 0)=\int_{\hat{X}} b(\hat{y}, x) k_{t}^{(1)}(\hat{y}) d y .
$$

Then by (4.1) we arrive at

$$
\begin{aligned}
k_{t}^{(1)}(x, 0)= & \int_{\mathbb{R}^{d}}\left(\int_{0}^{\infty} b\left(y, a_{y} \mid x\right) k_{t}^{(1)}\left(y, a_{y}\right) d a_{y}\right) d y \\
= & \int_{\mathbb{R}^{d}} \int_{0}^{t} b\left(y, a_{y} \mid x\right) k_{t-a_{y}}^{(1)}(y, 0) \\
& \times \exp \left(-\int_{0}^{a_{y}} m\left(y, a_{y}-\theta\right) d \theta\right) d a_{y} d y \\
& +\int_{\mathbb{R}^{d}} \int_{t}^{+\infty} b\left(y, a_{y} \mid x\right) k_{0}^{(1)}\left(y, a_{y}-t\right) \\
& \times \exp \left(-\int_{0}^{t} m\left(y, a_{y}-\theta\right) d \theta\right) d a_{y} d y .
\end{aligned}
$$

The second summand in (4.2) is bounded by

$$
\beta \operatorname{ess} \sup _{y \in \mathbb{R}^{d}} \int_{0}^{\infty} k_{0}^{(1)}(y, a) d a
$$

uniformly in $t$ and $x$. Set

$$
u_{t}(x)=k_{t}^{(1)}(x, 0) .
$$

Now (4.2) can be written in the following form

$$
u=A u+v,
$$

where

$$
(A u)_{t}(x)=\int_{\mathbb{R}^{d}} \int_{0}^{t} b\left(y, a_{y} \mid x\right) u_{t-a_{y}}(y) e^{-\int_{0}^{a_{y}} m\left(y, a_{y}-\theta\right) d \theta} d a_{y} d y,
$$




$$
v_{t}(x)=\int_{\mathbb{R}^{d}} \int_{t}^{+\infty} b\left(y, a_{y} \mid x\right) k_{0}^{(1)}\left(y, a_{y}-t\right) e^{-\int_{0}^{t} m\left(y, a_{y}-\theta\right) d \theta} d a_{y} d y .
$$

For a $C^{1}$-function $u: \mathbb{R}_{+} \rightarrow L^{\infty}\left(\mathbb{R}^{d}\right)$ and some $\alpha \geq 0$, we set

$$
\|u\|_{\alpha}=\sup _{t \geq 0} e^{-\alpha t}\left\|u_{t}\right\|_{L^{\infty}\left(\mathbb{R}^{d}\right)} .
$$

Let $\mathcal{U}_{\alpha}$ be the Banach space of such functions with norm as in (4.3). Then $A$ defined above is a bounded linear operator on $\mathcal{U}_{\alpha}$ with the norm satisfying

$$
\|A\|_{\alpha}=\beta \underset{y \in \mathbb{R}^{d}}{\operatorname{ess} \sup _{0}} \int_{0}^{\infty} e^{-\alpha a-\int_{0}^{a} m(y, \theta) d \theta} d a .
$$

For some $q \in(0,1)$, we can choose $\alpha$ such that $\|A\|_{\alpha} \leq q$. Then the solution of the equation above is

$$
u=(I-A)^{-1} v
$$

which allows us to obtain

$$
k_{t}^{(1)}(x, 0)=u_{t}(x)=(B v)_{t}(x):=\sum_{n=0}^{\infty}\left(A^{n} v\right)_{t}(x) .
$$

By means of (4.4) we get in (4.1)

$$
k_{t}^{(1)}(x, a)= \begin{cases}k_{0}{ }^{(1)}(x, a-t) \exp \left(-\int_{0}^{t} m(x, a-\theta) d \theta\right), & t \leq a \\ \sum_{n=0}^{\infty}\left(A^{n} v\right)_{t-a}(x) \exp \left(-\int_{0}^{a} m(x, a-\theta) d \theta\right), & t>a .\end{cases}
$$

4.2. The solution for $\boldsymbol{n}=\mathbf{2}$. Here we find $k_{t}{ }^{(2)}\left(y, a_{y}, x, a_{x}\right)$. Since it is supposed to be symmetric, we find it for $a_{x} \leq a_{y}$. By (3.7) we have

$$
\begin{aligned}
k_{t}^{(2)}\left(y, a_{y}, x, a_{x}\right)= & k_{0}{ }^{(2)}\left(\hat{\eta}_{t}\right) \\
& \times \exp \left(-\int_{0}^{t}\left(m\left(y, a_{y}-\theta\right)+m\left(x, a_{x}-\theta\right)\right) d \theta\right)
\end{aligned}
$$

when $t \leq a_{x}$ and

$$
\begin{aligned}
k_{t}{ }^{(2)}\left(y, a_{y}, x, a_{x}\right)= & k_{t-a_{x}}{ }^{(2)}\left(y, a_{y}-a_{x}, x, 0\right) \\
& \times \exp \left(-\int_{0}^{a_{x}}\left(m\left(y, a_{y}-\theta\right)+m\left(x, a_{x}-\theta\right)\right) d \theta\right)
\end{aligned}
$$

otherwise.

To find $k_{t-a_{x}}^{(2)}\left(y, a_{y}-a_{x}, x, 0\right)$ by $(3.3)$ we get

$$
\begin{aligned}
k_{t}^{(2)}(y, a, x, 0)= & \int_{\mathbb{R}^{d}} \int_{0}^{+\infty} b\left(z, a_{z} \mid x\right) k_{t}^{(2)}\left(y, a ; z, a_{z}\right) d a_{z} d z \\
& +k_{t}^{(1)}(y, a) b(y, a \mid x) .
\end{aligned}
$$


For $t \leq a$, we have in (4.8) the following. By (4.5) for $k_{t}^{(1)}(y, a)$, and (4.6), (4.7) for $k_{t}^{(2)}\left(y, a ; z, a_{z}\right)$, we have

$$
\begin{aligned}
k_{t}^{(2)}(y, a, x, 0)= & b(y, a \mid x) k_{0}^{(1)}(y, a-t) \exp \left(-\int_{0}^{t} m(y, a-\theta) d \theta\right) \\
& +\int_{\mathbb{R}^{d}} \int_{0}^{t} b\left(z, a_{z} \mid x\right) k_{t-a_{z}}^{(2)}\left(y, a-a_{z} ; z, 0\right) \\
& \times \exp \left(-\int_{0}^{a_{z}}\left(m\left(z, a_{z}\right)+m\left(y, a_{y}-\theta\right)\right) d \theta\right) d a_{z} d z \\
& +\int_{\mathbb{R}^{d}} \int_{t}^{+\infty} b\left(z, a_{z} \mid x\right) k_{0}^{(2)}\left(y, a-t ; z, a_{z}-t\right) \\
& \times \exp \left(-\int_{0}^{t}\left(m(y, a-\theta)+m\left(z, a_{z}-\theta\right)\right) d \theta\right) d a_{z} d z
\end{aligned}
$$

On the other hand, for $t>a$ we rewrite (4.8) as follows

$$
\begin{aligned}
k_{t}^{(2)}(y, a, x, 0)= & b(y, a \mid x)(B v)_{t-a}(y) \exp \left(-\int_{0}^{a} m(y, a-\theta) d \theta\right) \\
& +\int_{\mathbb{R}^{d}} \int_{0}^{a} b\left(z, a_{z} \mid x\right) k_{t-a_{z}}^{(2)}\left(y, a-a_{z} ; z, 0\right) \\
& \left.\times \exp \left(-\int_{0}^{a_{z}}\left(m\left(z, a_{z}-\theta\right)\right)+m(y, a-\theta)\right) d \theta\right) d a_{z} d z \\
& +\int_{\mathbb{R}^{d}} \int_{a}^{t} b\left(z, a_{z} \mid x\right) k_{t-a}^{(2)}\left(y, 0 ; z, a_{z}-a\right) \\
& \times \exp \left(-\int_{0}^{a}\left(m(y, a-\theta)+m\left(z, a_{z}-\theta\right)\right) d \theta\right) d a_{z} d z \\
& +\int_{\mathbb{R}^{d}} \int_{t}^{+\infty} b\left(z, a_{z} \mid x\right) k_{t-a}^{(2)}\left(y, 0 ; z, a_{z}-a\right) \\
& \times \exp \left(-\int_{0}^{a}\left(m(y, a-\theta)+m\left(z, a_{z}-\theta\right)\right) d \theta\right) d a_{z} d z .
\end{aligned}
$$

Now we solve (4.9) and (4.10) as a single equation in the space of functions $\mathbb{R}_{+} \ni t \mapsto w_{t} \in C^{1}\left(\mathbb{R}_{+}\right) \otimes L^{\infty}\left(\left(\mathbb{R}^{d}\right)^{2}\right) \otimes L^{1}\left(\mathbb{R}_{+}\right)$continuously differentiable with respect to $t$, essentially bounded and measurable with respect to $x, y$ and integrable with respect to $a$. By letting

$$
w_{t}(y, a, x)=k_{t}^{(2)}(y, a, x, 0)
$$

we obtain

$$
w_{t}(y, a, x)=\left(A_{2} w\right)_{t}(y, a, x)+f_{t}(y, a, x) .
$$


For $t \leq a$, define

$$
\begin{aligned}
\left(A_{2} w\right)_{t}(y, a, x)= & \int_{\mathbb{R}^{d}} \int_{0}^{t} b\left(z, a_{z} \mid x\right) w_{t-a_{z}}\left(z, y, a-a_{z}\right) \\
& \left.\times \exp \left(-\int_{0}^{a}(m(y, a-\theta))+m\left(z, a_{z}-\theta\right)\right) d \theta\right) d a_{z} d z, \\
f_{t}(y, a, x)= & \int_{\mathbb{R}^{d}} \int_{t}^{+\infty} b\left(z, a_{z} \mid x\right) k_{0}^{(2)}\left(y, a-t, z, a_{z}-t\right) \\
& \times \exp \left(-\int_{0}^{t}\left(m(y, a-\theta)+m\left(z, a_{z}-\theta\right)\right) d \theta\right) d a d y \\
& +k_{t}^{(1)}(y, a) b(x, y, a),
\end{aligned}
$$

and for $t>a$,

$$
\begin{aligned}
\left(A_{2} w\right)_{t}(y, a, x)= & \int_{\mathbb{R}^{d}} \int_{0}^{a} b\left(z, a_{z} \mid x\right) w_{t-a_{z}}\left(z, y, a-a_{z}\right) \\
& \left.\times \exp \left(-\int_{0}^{a_{z}}(m(y, a-\theta))+m\left(z, a_{z}-\theta\right)\right) d \theta\right) d a_{z} d z \\
& +\int_{\mathbb{R}^{d}} \int_{a}^{t} b\left(z, a_{z} \mid x\right) w_{t-a}\left(y, z, a_{z}-a\right) \\
& \left.\times \exp \left(-\int_{0}^{a}(m(y, a-\theta))+m\left(z, a_{z}-\theta\right)\right) d \theta\right) d a_{z} d z \\
& +\int_{\mathbb{R}^{d}} \int_{t}^{\infty} b\left(z, a_{z} \mid x\right) w_{t-a}\left(y, z, a_{z}-a\right) \\
& \left.\times \exp \left(-\int_{0}^{a}(m(y, a-\theta))+m\left(z, a_{z}-\theta\right)\right) d \theta\right) d a_{z} d z, \\
& f_{t}(y, a, x)=k_{t}^{(1)}(y, a) b(y, a, x) .
\end{aligned}
$$

Let $\mathcal{W}_{\alpha}$ be a space of functions $w_{t}$ with the norm

$$
\|w\|_{\alpha}=\sup _{t \geq 0} e^{-\alpha t} \operatorname{ess~sup}_{(x, y) \in\left(\mathbb{R}^{d}\right)^{2}} \int_{0}^{\infty}\left|w_{t}(y, a, x)\right| d a .
$$

It is clear that

$$
\int_{0}^{\infty}\left(A_{2} w\right)_{t}(y, a, x) d a=\int_{0}^{t}\left(A_{2} w\right)_{t}(y, a, x) d a+\int_{t}^{\infty}\left(A_{2} w\right)_{t}(y, a, x) d a .
$$

Therefore,

$$
\begin{aligned}
\int_{0}^{\infty}\left(A_{2} w\right)_{t}(y, a, x) & d a=\int_{0}^{t} \int_{\mathbb{R}^{d}} \int_{0}^{a} b\left(z, a_{z} \mid x\right) w_{t-a_{z}}\left(z, y, a-a_{z}\right) \\
\times & \exp \left(-\int_{0}^{a_{z}}\left(m(y, a-\theta)+m\left(z, a_{z}-\theta\right)\right) d \theta\right) d a_{z} d z d a
\end{aligned}
$$




$$
\begin{aligned}
& +\int_{0}^{t} \int_{\mathbb{R}^{d}} \int_{a}^{t} b\left(z, a_{z} \mid x\right) w_{t-a}\left(y, z, a_{z}-a\right) \\
& \times \exp \left(-\int_{0}^{a}\left(m(y, a-\theta)+m\left(z, a_{z}-\theta\right)\right) d \theta\right) d a_{z} d z d a \\
& +\int_{0}^{t} \int_{\mathbb{R}^{d}} \int_{t}^{\infty} b\left(z, a_{z} \mid x\right) w_{t-a}\left(y, z, a_{z}-a\right) \\
& \times \exp \left(-\int_{0}^{a}\left(m(y, a-\theta)+m\left(z, a_{z}-\theta\right)\right) d \theta\right) d a_{z} d z d a \\
& +\int_{t}^{\infty} \int_{\mathbb{R}^{d}} \int_{0}^{t} b\left(z, a_{z} \mid x\right) w_{t-a_{z}}\left(z, y, a-a_{z}\right) \\
& \times \exp \left(-\int_{0}^{a}\left(m(y, a-\theta)+m\left(z, a_{z}-\theta\right)\right) d \theta\right) d a_{z} d z d a .
\end{aligned}
$$

To estimate a value of the previous integrate we use (3.2) and the fact that $e^{-a}<1$, where $a>0$. Let $\alpha=\frac{\beta}{q}$, for some fixed $q<1$, therefore

$$
\left\|A_{2} w\right\|_{\alpha} \leq q\|w\|_{\alpha} .
$$

We can write the solution for (4.11) as

$$
k_{t}(x, 0, y, a)=w_{t}(x, y, a)=\sum_{n=0}^{\infty}\left(A_{2}{ }^{n} f\right)_{t}(x, y, a) .
$$

The solution for $k^{(2)}\left(x, a_{x}, y, a_{y}\right)$ takes the form: for $t \leq a_{x} \leq a_{y}$

$$
\begin{aligned}
k^{(2)}\left(x, a_{x}, y, a_{y}\right)= & k_{0}^{(2)}\left(x, a_{x}-t ; y, a_{y}-t\right) \\
& \times \exp \left(-\int_{0}^{t}\left(m\left(x, a_{x}-\theta\right)+m\left(y, a_{y}-\theta\right)\right) d \theta\right),
\end{aligned}
$$

for $a_{x} \leq t$

$$
\begin{aligned}
k^{(2)}\left(x, a_{x}, y, a_{y}\right)= & \left(\sum_{n=0}^{\infty}\left(A_{2}{ }^{n} f\right)_{t-a_{x}}\left(y, a_{y}-a_{x}, x\right)+k_{t-a_{x}}^{(1)}(y, a) b\left(y, a_{y} \mid x\right)\right) \\
& \times \exp \left(-\int_{0}^{a_{x}}\left(m\left(x, a_{x}-\theta\right)+m\left(y, a_{y}-\theta\right)\right) d \theta\right) .
\end{aligned}
$$

For $a_{x}<t \leq a_{y}$ with an appropriate formula for $f_{t}(4.12)$ :

$$
\begin{aligned}
\left(A_{2} f\right)_{t}(y, a, x)= & \int_{\mathbb{R}^{d}} \int_{0}^{t} b\left(z, a_{z} \mid x\right) f_{t-a_{z}}\left(z, y, a-a_{z}\right) \\
& \left.\times \exp \left(-\int_{0}^{a}(m(y, a-\theta))+m\left(z, a_{z}-\theta\right)\right) d \theta\right) d a_{z} d z .
\end{aligned}
$$


For $a_{x} \leq a_{y}<t, f_{t}$ like in (4.13):

$$
\begin{aligned}
\left(A_{2} f\right)_{t}(y, a, x)= & \int_{\mathbb{R}^{d}} \int_{0}^{a} b\left(z, a_{z} \mid x\right) f_{t-a_{z}}\left(z, y, a-a_{z}\right) \\
& \left.\times \exp \left(-\int_{0}^{a_{z}}(m(y, a-\theta))+m\left(z, a_{z}-\theta\right)\right) d \theta\right) d a_{z} d z \\
& +\int_{\mathbb{R}^{d}} \int_{a}^{t} b\left(z, a_{z} \mid x\right) f_{t-a}\left(y, z, a_{z}-a\right) \\
& \left.\left.\times \exp \left(-\int_{0}^{a}(m(y, a-\theta))+m\left(z, a_{z}-\theta\right)\right) d \theta\right)\right) d a_{z} d z \\
& +\int_{\mathbb{R}^{d}} \int_{t}^{\infty} b\left(z, a_{z} \mid x\right) f_{t-a}\left(y, z, a_{z}-a\right) \\
& \left.\times \exp \left(-\int_{0}^{a}(m(y, a-\theta))+m\left(z, a_{z}-\theta\right)\right) d \theta\right) d a_{z} d z .
\end{aligned}
$$

The solution for these two correlation functions was presented to show how they can look like explicitly and make them easier to imagine. Moreover, we prepare background to further calculations.

If we have the formula for all correlation functions we can come back to equation (2.1) and prove that the solution for this equation exists and keep required assumptions.

5. Acknowledgement. The author would like to thank the Bielefeld University and all participants of the project No 57154469 "Dynamics of Large Systems of Interacting Entities" supported by the DAAD.

\section{REFERENCES}

[1] Berns, Ch., Kondratiev, Y., Kozitsky, Y., Kutoviy, O., Kawasaki dynamics in continuum: micro- and mesoscopic descriptions, J. Dynam. Differential Equations 25 (4) (2013), 1027-1056.

[2] Bogoliubov, N., Problems of a Dynamical Theory in Statistical Physics, Gostekhisdat, Moscow, 1946 (in Russian). English translation, in: J. de Boer and G. E. Uhlenbeck (editors), Studies in Statistical Mechanics, Volume 1, 1-118, North-Holland, Amsterdam, 1962.

[3] Daletskii, A., Kondratiev, Y., Kozitsky, Y., Phase transitions in continuum ferromagnets with unbounded spins, J. Math. Phys. 56 (11) (2015), 1-20.

[4] Finkelshtein, D., Kondratiev, Y., Oliveira, M., Markov evolutions and hierarchical equations in the continuum I. One-component systems, J. Evol. Equ. 9 (2) (2009), $197-233$.

[5] Iannelli, M., Mathematical theory of age-structured population dynamics, Applied Mathematics Monographs, Giardini Editori e Stampatori, Pisa, 1995.

[6] Kondratiev, Y., Kuna, T., Harmonic analysis on configuration space. I. General theory, Infin. Dimens. Anal. Quantum Probab. Relat. Top. 5 (2) (2002), 201-233.

[7] Kondratiev, Y., Kutoviy, O., Pirogrov, S., Correlation functions and invariant measures in continuous contact model, Infin. Dimens. Anal. Quantum Probab. Relat. Top. 11 (2) (2008), 231-258. 
[8] Kondratiev, Y., Lytvynov, E., Us, G., Analysis and geometry on $\mathbb{R}_{+}$marked configuration spaces, Meth. Func. Anal. and Geometry 5 (1) (2006), 29-64.

[9] Méléard, S., Tran, V., Trait substitution sequence process and canonical equation for age-structured populations, J. Math. Biol. 58 (6) (2009), 881-921.

[10] Méléard, S., Tran, V., Slow and fast scales for superprocess limits of age-structured populations, Stochastic Process. Appl. 122 (1) (2012), 250-276.

[11] Minlos, R. A., Lectures on statistical physics, Russian Mathematical Surveys 23 (1) (1968), 133-190

[12] Tanaś, A., A continuum individual based model of fragmentation: dynamics of correlation functions, Ann. Univ. Mariae Curie-Skłodowska Sect. A 64 (2) (2015), 73-83.

Dominika Jasińska

Institute of Mathematics

Maria Curie-Skłodowska University

pl. M. Curie-Skłodowskiej 1

20-031 Lublin

Poland

e-mail: jasdominika@wp.pl

Received November 15, 2016 